\title{
Importance of wavenumber dependence of Biot numbers in one-sided models of evaporative Marangoni instability: horizontal layer and spherical droplet
}

\author{
H. Machrafi* \\ Université de Liège, Institut de Physique B5a, \\ Allée du 6 Août, 17, B-4000 Liège 1, Belgium \\ A. Rednikov and P. Colinet \\ Université Libre de Bruxelles, TIPs - Fluid Physics, CP165/67, \\ Avenue F.D. Roosevelt, 50, B-1050 Bruxelles, Belgium \\ PC. Dauby \\ Université de Liège, Institut de Physique B5a, \\ Allée du 6 Août, 17, B-4000 Liège 1, Belgium
}

\begin{abstract}
A one-sided model of the thermal Marangoni instability owing to evaporation into inert gas is developed. Two configurations are studied in parallel: a horizontal liquid layer and a spherical droplet. With the dynamic gas properties being admittedly negligible, one-sided approaches typically hinge upon quantifying heat/mass transfer through the gas phase by means of transfer coefficients (like in the Newton's cooling law), which in dimensionless terms eventually corresponds to the Biot number. Quite a typical arrangement encountered in the literature is a constant Biot number, the same for perturbations of different wavelengths and maybe even the same as for the reference state. In the present work, we underscore the relevance of accounting for its wavenumber dependence, which is especially the case in the evaporative context with relatively large values of the resulting effective Biot number. We illustrate the effect in the framework of the Marangoni instability thresholds. As a concrete example, we consider HFE-7100 (a standard refrigerant) for the liquid and air for the inert
\end{abstract} gas.

*H.Machrafi@ulg.ac.be 


\section{INTRODUCTION}

When a vertical temperature gradient is imposed across a horizontal liquid layer, its horizontally uniform state can become convectively unstable. This is generally known as a Bénard instability. This instability can essentially be driven either by buoyancy (Rayleigh instability) or by surface-tension gradients (Marangoni instability). This latter instability mechanism was first pointed out in [1], and since then has become a key effect in the studies of the Bénard instability [2],[3],[4] in the presence of a free interface. Rather than by an externally imposed temperature gradient, Bénard instability can also be caused by evaporation, when the liquid evaporates into an inert gas right above it. Due to the latent heat of evaporation, this leads to a reduction of the temperature at the surface of the liquid. Thus, as such, vertical temperature gradients occur naturally inside the system (even in isothermal surroundings), e.g. [5], [6]. Evaporation is therefore potentially able to induce thermal Rayleigh-Marangoni-Bénard instabilities, which has indeed been observed experimentally and numerically [7], [8], [9], [10], [11]. From the viewpoint of a critical layer thickness for the instability onset (the issue we shall focus upon in the present paper), the Marangoni effect is often the dominating instability mechanism. Indeed, the critical thicknesses prove to be rather small for reasonably volatile liquids [12], and the buoyancy effects are expected to play no role there. Therefore, it is only the Marangoni instability that will be considered in this paper and used for further interpretations. Apart from evaporating horizontal layers, we shall here also be interested in a similar problem of the Marangoni instability in evaporating spherical droplets. The critical droplet radii are likewise expected to be small, so that gravity effects are not essential. Such a spherical droplet problem, formally posed in the absence of gravity, would at the very least correspond to droplets in microgravity.

The onset of instability is linked to physical properties and competing time scales in both the liquid and gas phases. Often, the density and dynamic viscosity in the gas are small and, besides, the diffusive time scales in the gas phase are small with respect to those in the liquid phase. Therefore, the roles of the two phases in the instability mechanisms can be very different and often the gas phase plays a more passive one. For this reason, the gas layer is often not studied in detail and a one-sided model of the system is rather invoked, for which the influence of the gas on the liquid is described through the introduction of phenomenological boundary conditions at the liquid upper surface. A well-known example of such a phenomenological relationship is the Newton's law of cooling, which states that the heat flux at the liquid upper surface is proportional, by means of a certain transfer coefficient, to the difference between the temperature of this surface and the temperature far away in the gas. The Biot number is essentially a dimensionless version of this transfer coefficient. We note that in this way the same Biot number equally applies both to the base (horizontally homogeneous, reference) state of the system and to perturbations. In other words, it is constant (wavenumber-independent). This kind of approach to describe the gas influence has been used quite often in the literature, both for horizontal layers [13], [14], [15] and for spherical droplets [16]. In the case of evaporation (into ambient air), one arrives again at a single constant effective thermal Biot number, now dependent on both mass and heat transfer coefficients in the gas [17]. On the other hand, no heuristic transfer coefficients and Biot numbers are adopted in the studies [18], [19] for evaporating spherical droplets. Rather, a Biot number is deduced from an exact treatment of the spherically symmetric heat and mass transfer problem in the gas. Yet, this same Biot number (up to linearizations) is 
subsequently applied to the problem of perturbations, hence once again implying a constant (wavenumber-independent) Biot number.

Another type of Biot number obtained during the reduction of two-layer systems to onesided ones, is dependent on the wavenumber of perturbations and was introduced for instance in [5], [6], [12], [20], [21]. Considering the wavenumber dependence of the Biot number is physically more consistent, since this implies taking into account transfer through the gas phase not only between the interface and the ambient as for the former (constant) type of Biot numbers, but also between different interface spots. Overall, a wavenumber-dependent Biot number is substantially different from constant ones, e.g. because it renders the problem non-local. So, a question arises whether considering the wavenumber dependence in the Biot number is of any practical importance. In the present work, it is therefore the purpose to explore this question, which we shall do in the context of the Marangoni-instability onset. A linear stability analysis will be carried out comparing the results based on the constant and the wavenumber-dependent Biot numbers. With the intention to generalize this scope, we pursue the study for both horizontal layers and spherical droplets.

It is clear that in such evaporating configurations, the reference state, upon which development of the Marangoni instability is considered, is inherently transient. After all, neither the layer thickness, nor the droplet radius remain constant in time. However, assuming evaporation to be slow compared to the thermal time scale in the liquid, which we shall legitimately do here, we arrive at a quasi-stationary formulation with respect to the size of the system, treated as constant. Yet the reference state can still be transient with respect to a possible evolution of the temperature and concentration fields from their initial values, although stationary profiles thereof are eventually attained. Furthermore, in the droplet case, it is only at this transient stage that an essential Marangoni instability can in fact be expected, when there exists a non-trivial temperature profile inside the droplet [18], which becomes merely constant when attaining stationarity [22]. The same actually goes for the layer with an insulated bottom, which is a close counterpart of the droplet in this regard. Thus, in the present paper, we generally deal with transient reference states, even if the variation of the size of the system is neglected. The stability analysis is then carried out by means of the frozen-time approach. In contrast, note that for the layer with a fixed temperature at the bottom, a non-trivial stationary reference state is attained and the Marangoni instability can exist at this stage too.

The paper is organized as follows. In section II, we continue with describing the configurations of the horizontal layer and the spherical droplet, as well as the basic assumptions. These two configurations will be treated side by side throughout the paper. In section III, the reference state is considered. Section IV is dedicated to the linear stability analysis, a first step of which is a derivation of the wavenumber-dependent Biot number. The results are discussed in section $\mathrm{V}$, and the conclusions are summarized in section VI.

\section{PHYSICAL DESCRIPTION OF THE SYSTEMS}

The two evaporative systems that are considered in this paper are schematically represented in Fig.1, namely a horizontal layer with an undeformable free surface and a spherical droplet (for which gravity is neglected), respectively. In both these systems, we have a pure liquid (here we consider the example of HFE-7100, a common refrigerant commercialized by 3M) evaporating into ambient air maintained at $T_{a m b}, p_{a m b}$ and $\chi_{a m b}$, which are the ambient temperature, pressure and vapor molar fraction (ambient humidity), respectively. Thus, 

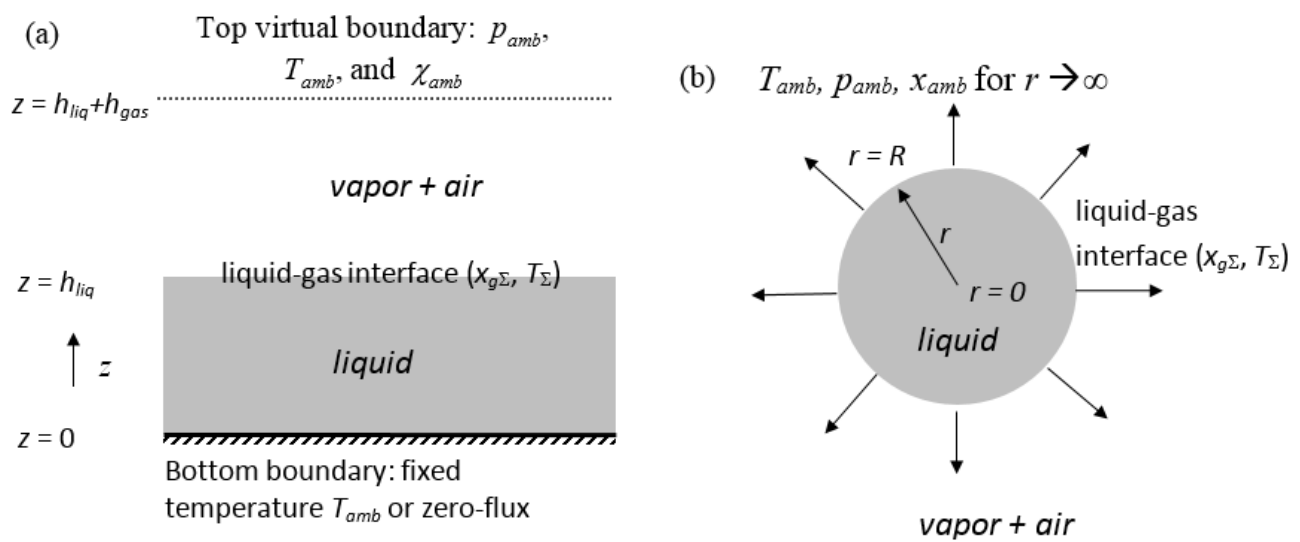

FIG. 1. (Color online) Sketches of the horizontal-layer (a) and spherical-droplet (b) systems.

the gas phase is composed of air (whose solvability in the liquid is neglected) and vapor. In this work, we shall consider $\chi_{a m b}=0$. The aforementioned ambient conditions are formally imposed infinitely far from the droplet, whereas in the layer configuration they are imposed at a certain transfer distance $h_{\text {gas }}$ from the interface. This transfer distance can be described as a typical equivalent (effective) diffusion length in the gas phase at which the diffusive transport is formally of the same magnitude as the convective transport in a real setup, as determined by air currents which may be naturally present (e.g., due to buoyancy) or deliberately created (ventilation or gas flow, see [20]). In some cases, it can be roughly identified with the height of an open container [12]. At the bottom of the liquid layer, we consider two types of conditions: an insulated bottom and a bottom with a fixed temperature equal to $T_{a m b}$. The insulated-bottom situation is a closer counterpart of the droplet case. The quantities at the liquid-gas interface are marked by the subscript ' $\Sigma$ ', e.g. $T_{\Sigma}$ and $\chi_{g \Sigma}$ for the interfacial temperature and vapor molar fraction, respectively. Local thermodynamic equilibrium is assumed at the interface, so that $\chi_{g \Sigma}$ is determined by the saturation conditions. Both systems defined possess a symmetry: a horizontal translational one for the layer and a spherical (rotational) one for the droplet. The base (reference) states of both systems clearly respect these symmetries: everything depends just on the vertical coordinate $z$ for the layer, and on the radial coordinate $r$ for the droplet. Evaporation (provided that $\left.\chi_{g \Sigma}>\chi_{a m b}\right)$ then leads to the cooling of the liquid-gas interface $\left(T_{\Sigma}<T_{a m b}\right)$ for both systems. Marangoni instability is then expected to set in for a sufficiently thick layer or large droplet, leading to a symmetry breakup. It is this Marangoni instability onset that we are concerned with in the present paper.

The horizontal layer is characterized by a liquid thickness $h_{l i q}$, while the spherical droplet by a droplet radius $R$. For convenience, we define a generic symbol, $\ell$, for the characteristic length:

$$
\ell \equiv \begin{cases}h_{l i q} & \text { (layer }) \\ R & \text { (droplet) }\end{cases}
$$

Note that the transfer distance yields, for the horizontal layer, an extra parameter $h_{\text {gas }}$ with respect to the spherical droplet. However, as far as this extra parameter is concerned, we shall rather work in terms of a dimensionless total height $H \equiv\left(h_{\text {liq }}+h_{g a s}\right) / h_{\text {liq }}$. We will 
show later on in this paper that, despite the "infinite gas layer" of the droplet, a qualitative correspondence with the droplet system is observed for $H-1=O(1)$, whereas the cases $H-1 \ll 1$ and $H \gg 1$ may be essentially different.

The other noteworthy assumptions made in the analysis are the following. We assume that the evaporation process is slow with respect to the thermal diffusion time in the liquid. In other words, the time scale of the interface regression $\ell /(d \ell / d t)$ is much larger than the heat diffusion time scale $\ell^{2} / \kappa_{l}$, which is equivalent to the Péclet number $\ell(d \ell / d t) / \kappa_{l}$ being small. Here, $t$ is the time, $\kappa$ is the thermal diffusivity, while the subscripts ' $l$ ' and ' $g$ ' at the material properties refer to the liquid and gas phases, respectively. The implication of this assumption is that the interface regression can be neglected, and the layer thickness and the droplet radius treated as constant (quasi-stationarity) when studying the development of the reference profiles (generally assumed transient) and of the Marangoni instability. Likewise, in a regime of Marangoni convection, the contribution of evaporation to the velocity field in the liquid is negligible against the Marangoni-induced velocity field. Furthermore, on account of the generally satisfied inequalities $\kappa_{l} \ll \kappa_{g}$ and $\kappa_{l} \ll D_{g}$, where $D$ is the diffusion coefficient, the characteristic times in the gas will be much smaller than the thermal time in the liquid so that we can assume quasi-stationarity in the gas phase, while keeping transience in the liquid. The inequalities $\kappa_{l} \ll \kappa_{g}$ and $\kappa_{l} \ll D_{g}$ mean also that for the instability or convection developing in the liquid, we can neglect convective effects (with respect to the diffusional ones) in the gas phase even when they are essential in the liquid. Possible buoyancy convection in the gas does not appear to be an issue either, given a stable stratification therein due to the interface being colder than the ambient medium (evaporative cooling) and the vapor being in most cases heavier than air (as for our example with HFE7100). All these observations, together with the fact that the gas dynamic viscosity $\mu_{g}$ is negligible against the liquid one $\mu_{l}$, encourage the use of a one-sided model for our study, with the gas phase being accounted for by an appropriate Biot number. We will show that it is in fact important that this Biot number be defined as a function of the wavenumber.

It should also be mentioned that convective effects in the gas phase are not only associated with Marangoni convection, but also with the velocity due to evaporation (Stefan flow). The convective effect of Stefan flow is inessential for dilute vapors, but becomes important if the vapor content is comparable with that of the inert gas. The same goes for the effect of gas-density non-uniformity. In the present paper, we shall formally work in the dilute-vapor limit and thus have a quasi-stationary pure-diffusion regime for mass transfer in the gas phase of constant density, hence simply $\nabla^{2} \chi_{g}=0$ ( $\nabla^{2}$ being the laplacian $)$ in terms of the vapor molar fraction $\chi_{g}$ (see [25] and [26]). As $\kappa_{g}$ and $D_{g}$ are typically of the same order for gases, for the gas temperature field $T_{g}$ we also have simply $\nabla^{2} T_{g}=0$.

\section{REFERENCE STATE}

In the present section, we consider the reference (base) states of the two systems involved, whose linear stability will subsequently be studied. In the gas phase, the analysis can right away be performed analytically thanks to the hypotheses outlined in the previous section. This is what forms the subject of subsection III A, which is then made use of in subsection III B in order to arrive at a one-sided formulation in the liquid. Finally, the computed timedependent reference profiles in the liquid are presented in subsection III C. Hereafter, the subscript 'ref' will be used to mark the dependent variables of the reference state. 


\section{A. Gas phase}

With the hypotheses described before and for the layer and the droplet configurations, respectively, the reference molar-fraction and temperature fields in the gas are simply

$$
\chi_{g, r e f}=\left\{\begin{array}{c}
\left.\chi_{g \Sigma, r e f}-\left(\chi_{g \Sigma, r e f}-\chi_{a m b}\right) \frac{z-h_{l i q}}{h_{g a s}} \quad \text { (layer }\right) \\
\chi_{a m b}-\left(\chi_{a m b}-\chi_{g \Sigma, r e f}\right) \frac{R}{r} \quad \text { (droplet) }
\end{array}\right.
$$

and

$$
T_{g, r e f}=\left\{\begin{array}{c}
T_{\Sigma, r e f}-\left(T_{\Sigma, r e f}-T_{a m b}\right) \frac{z-h_{l i q}}{h_{g a s}} \quad \text { (layer) } \\
T_{a m b}-\left(T_{a m b}-T_{\Sigma, r e f}\right) \frac{R}{r} \quad \text { (droplet) }
\end{array}\right.
$$

where the interface values $\chi_{g \Sigma, \text { ref }}$ and $T_{\Sigma, \text { ref }}$ are unknowns of the problem. They are related by $\chi_{g \Sigma, r e f}=p_{s a t}\left(T_{\Sigma, r e f}\right) / p_{a m b}$, where $p_{\text {sat }}$ is the saturation (vapor) pressure as a known function of temperature. Assuming ideal gases and using the Clausius-Clapeyron relation, we obtain

$$
\chi_{g \Sigma, r e f}=\frac{p_{s a t}\left(T_{a m b}\right)}{p_{a m b}} e^{-\frac{L M}{R g}\left(\frac{1}{T_{\Sigma, r e f}}-\frac{1}{T_{a m b}}\right)}
$$

where $p_{\text {sat }}\left(T_{a m b}\right)$ is considered known, $L$ is the latent heat of evaporation, $M$ is the molar mass of our liquid, and $R_{g}$ is the universal gas constant. With (2) and (3), the normal gradients at the interface, which will be needed later on, can be expressed as

$$
\begin{aligned}
& \left.\frac{\partial \chi_{g, r e f}}{\partial \mathbf{n}}\right|_{\Sigma}=\frac{\chi_{a m b}-\chi_{g \Sigma, r e f}}{\ell \delta_{g}} \\
& \left.\frac{\partial T_{g, r e f}}{\partial \mathbf{n}}\right|_{\Sigma}=\frac{T_{a m b}-T_{\Sigma, r e f}}{\ell \delta_{g}}
\end{aligned}
$$

for both configurations, where a unifying notation

$$
\delta_{g} \equiv\left\{\begin{array}{c}
H-1 \text { (layer) } \\
1 \text { (droplet) }
\end{array}\right.
$$

has been introduced in addition to (1) for convenience. Note therefore that it is apparently for $H=2$ that the biggest quantitative correspondence for the reference solutions between the two configurations can be expected. Also note that 


$$
\frac{\partial}{\partial \mathbf{n}} \equiv\left\{\begin{array}{cc}
\partial / \partial z & \text { (layer) } \\
\partial / \partial r & \text { (droplet) }
\end{array}\right.
$$

The evaporation mass flux $J\left(k g / \mathrm{m}^{2} s\right)$ or its molar counterpart $J / M$ is a sum of a convective (Stefan flow) and a diffusional part [20] (see also [27]). One has

$$
\frac{J}{M}=\frac{J}{M} \chi_{g \Sigma}-\left.n_{g} D_{g} \frac{\partial \chi_{g}}{\partial \mathbf{n}}\right|_{\Sigma}
$$

where $n_{g}=p_{a m b} /\left(R_{g} T_{a m b}\right)$ (assuming ideal gases) is the gas molar density (assumed constant throughout the gas and evaluated at ambient pressure and temperature). Thus,

$$
J=-\left.\frac{p_{a m b} D_{g} M}{R_{g} T_{a m b}\left(1-\chi_{g \Sigma}\right)} \frac{\partial \chi_{g}}{\partial \mathbf{n}}\right|_{\Sigma}
$$

Using Eq. (5), this yields the following result for the reference state evaporation flux:

$$
J_{r e f}=\frac{p_{a m b} D_{g} M}{R_{g} T_{a m b}} \frac{\chi_{g \Sigma, r e f}-\chi_{a m b}}{1-\chi_{g \Sigma, r e f}} \frac{1}{\ell \delta_{g}}
$$

for both configurations.

Overall, in the framework of the present analysis in the gas phase, there actually remains one undetermined quantity. Indeed, for the three interface quantities $J_{r e f}, \chi_{g \Sigma, r e f}$ and $T_{\Sigma, r e f}$, there are just two equations, Eqs. (4) and (11). Further progress can only be made by considering the liquid phase.

\section{B. Liquid phase}

In the liquid, the transient reference profile satisfies

$$
\frac{\partial T_{l, r e f}}{\partial t}=\kappa_{l} \nabla^{2} T_{l, r e f}
$$

where $\nabla^{2}=\partial^{2} / \partial z^{2}$ for the layer, and $\nabla^{2}=\partial^{2} / \partial r^{2}+(2 / r) \partial / \partial r$ for the droplet.

At the liquid-gas interface, we have the following boundary conditions:

$$
T_{l, r e f}=T_{\Sigma, r e f}
$$

and

$$
-\lambda_{l} \frac{\partial T_{l, r e f}}{\partial \mathbf{n}}+\lambda_{g} \frac{T_{a m b}-T_{\Sigma, r e f}}{\ell \delta_{g}}=L J_{r e f}
$$


The latter expresses the energy balance at the interface $-\lambda_{l} \partial T_{l} / \partial \mathbf{n}+\lambda_{g} \partial T_{g} / \partial \mathbf{n}=J L$, for the reference state, in which (6) has been introduced.

At the opposite end of the liquid domain ( $z=0$ for the layer and $r=0$ for the droplet), we impose

$$
\frac{\partial T_{l, r e f}}{\partial \mathbf{n}}=0
$$

implying an insulated bottom of the layer and no heat source in the center of the droplet (for the spherically symmetric reference profile).

For the layer, however, we shall also consider an important alternative setup, with the bottom temperature fixed at the ambient value:

$$
T_{l, r e f}=T_{a m b} \quad \text { (layer only) }
$$

at $z=0$.

The initial condition expresses that the temperature is everywhere equal to its ambient value:

$$
T_{l, r e f}=T_{a m b}
$$

at $t=0$.

Thus, the problem for the liquid temperature reference profile finally reduces to Eq. (12), with the interface conditions (4), (11), (13) and (14), bottom/center conditions (15) or (16), and the initial condition (17). Note that it has actually been reduced to a one-sided problem, owing to the large value of diffusivities in the gas phase (as discussed in section II). The solution is obtained numerically and the results are presented in the next subsection.

Before proceeding to the results, the following remark is made. The system of interface conditions (4), (11), (13) and (14) is non-linear with respect to $T_{\Sigma, r e f}$. However, a linearization can in principle be made under the assumption that the interface temperature remains close enough to $T_{a m b}$. In this way, the interface conditions can readily be reduced to a single one,

$$
\frac{\partial T_{l, r e f}}{\partial \mathbf{n}}+\frac{B i_{0, a m b}}{\ell}\left(T_{l, r e f}-T_{a m b}\right)=-\frac{L}{\lambda_{l}} J_{a m b}
$$

where

$$
J_{a m b}=\frac{p_{a m b} D_{g} M}{R_{g} T_{a m b}} \frac{p_{s a t}\left(T_{a m b}\right) / p_{a m b}-\chi_{a m b}}{1-p_{s a t}\left(T_{a m b}\right) / p_{a m b}} \frac{1}{\ell \delta_{g}}
$$

is the evaporation flux that would take place if the interface were exactly at the ambient temperature, and 


$$
B i_{0, a m b}=\frac{1}{\delta_{g}}\left\{\frac{\lambda_{g}}{\lambda_{l}}+\frac{L^{2} M^{2} D_{g}}{R_{g}^{2} T_{a m b}^{3} \lambda_{l}} \frac{p_{\text {sat }}\left(T_{a m b}\right)}{1-p_{s a t}\left(T_{a m b}\right) / p_{a m b}}\right\}
$$

is a Biot number (see [28]). Even though we here prefer not to use the linearized version (18) when solving for the reference state, the expression for the Biot number (20) is important for our purposes in the present paper. Indeed, an Ansatz similar to (18)-(20) is often encountered in the literature (e.g. [17], [29]), in the case when mass/heat exchange with the gas phase is modeled by means of Newton's law of cooling. However, the problem is that this same constant Biot number subsequently appears in the analysis of perturbations as well, whereas our goal in the present paper is to show that the Biot number for perturbations should actually be different and wavenumber-dependent.

Finally, note that in what follows we shall also use dimensionless versions of the variables $t, z, r$ and $T_{l}$, which in term of their dimensional counterparts are defined as $t \kappa_{l} / \ell^{2}, z / \ell$, $r / \ell$ and $\left(T_{l}-T_{a m b}\right) / \theta_{T}$, respectively, where $\theta_{T} \equiv J_{a m b} L \ell / \lambda_{l}$ is the temperature scale (while $\ell^{2} / \kappa_{l}$ and $\ell$ are obviously used as the time and length scales, respectively).

\section{Numerical solution for the transient reference profiles}

The problem formulated in the previous subsection is here solved for a concrete example with HFE-7100 as the liquid and air as the gas. Furthermore, we choose $T_{a m b}=298.15 \mathrm{~K}$, $p_{a m b}=1 \mathrm{~atm}$ and $\chi_{a m b}=0$. The physical properties of HFE-7100 can be found in Table I, for which we use those at the ambient conditions. Hence, the properties of the gas phase are just taken to be those of air $\left(\chi_{a m b}=0\right)$. The diffusion coefficient $D_{g}$ of HFE-7100 vapor in the gas is calculated using a correlation from [30] (see [20] for more details).

TABLE I. Physical properties of the system (HFE-7100+air) at $T_{a m b}=298.15 \mathrm{~K}, p_{a m b}=1 \mathrm{~atm}$, $\chi_{a m b}=0$.

\begin{tabular}{cc}
\hline \hline Physical property & Value \\
\hline$\lambda_{g}(\mathrm{~W} / \mathrm{Km})$ & $2.62 \times 10^{-2}$ \\
$D_{g}\left(\mathrm{~m}^{2} / \mathrm{s}\right)$ & $6.98 \times 10^{-6}$ \\
$\rho_{l}\left(\mathrm{~kg} / \mathrm{m}^{3}\right)$ & $1.482 \times 10^{3}$ \\
$\lambda_{l}(\mathrm{~W} / \mathrm{Km})$ & $6.9 \times 10^{-2}$ \\
$\kappa_{l}\left(\mathrm{~m}^{2} / \mathrm{s}\right)$ & $3.94 \times 10^{-8}$ \\
$\mu_{l}(\mathrm{Pas})$ & $5.8 \times 10^{-4}$ \\
$\gamma_{T}(\mathrm{~N} / \mathrm{Km})$ & $1.14 \times 10^{-4}$ \\
$p_{\text {sat }}(\mathrm{atm})$ & $2.65 \times 10^{-1}$ \\
$L(\mathrm{~J} / \mathrm{kg})$ & $1.116 \times 10^{5}$ \\
\hline \hline
\end{tabular}

In the stability analysis we are mostly interested in the temperature reference profiles in the liquid (which is the driving factor of the Marangoni instability). These profiles are presented in Figs. 2 and 3. The results for the horizontal layer are shown in Fig. 2 for $H$ values of 2, 11 and 101 both for the zero-flux and for fixed-temperature boundary conditions at the bottom. Fig. 3 shows the corresponding results for the spherical droplet. 


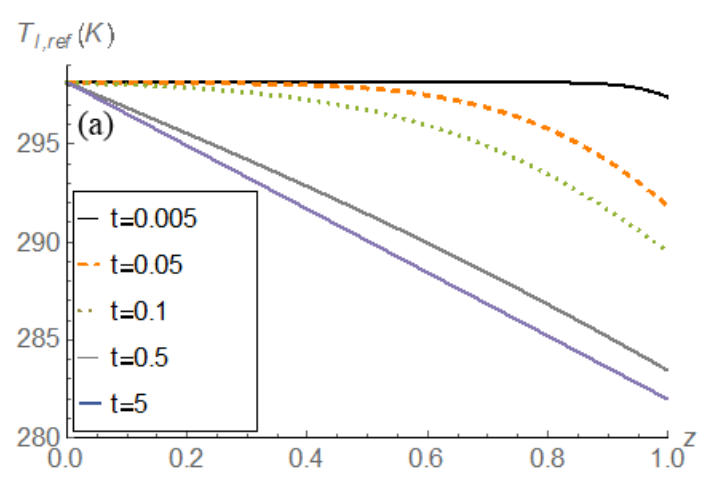

$$
T_{1, \text { ref }}(K)
$$
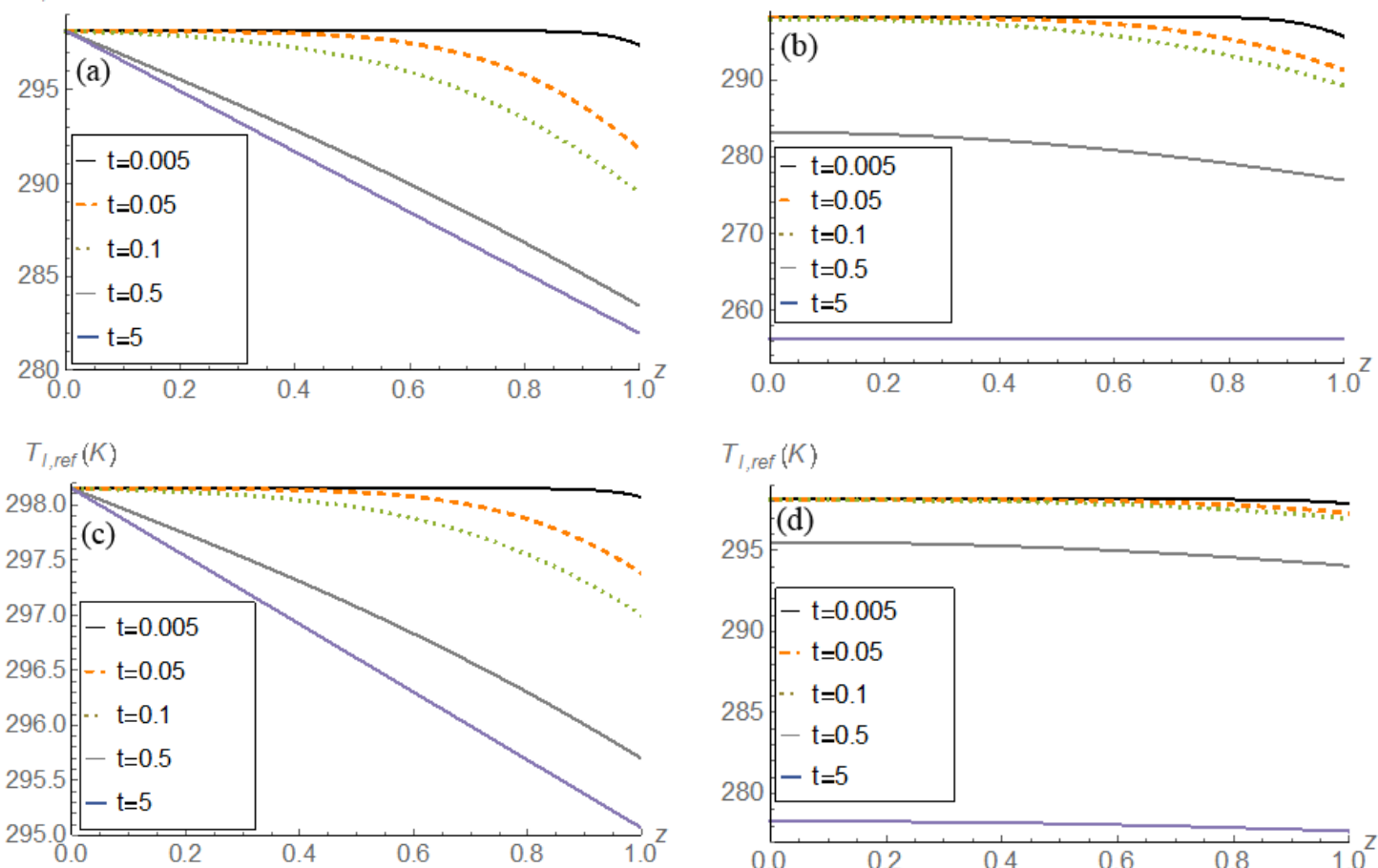

$$
T_{l, \text { ref }}(K)
$$
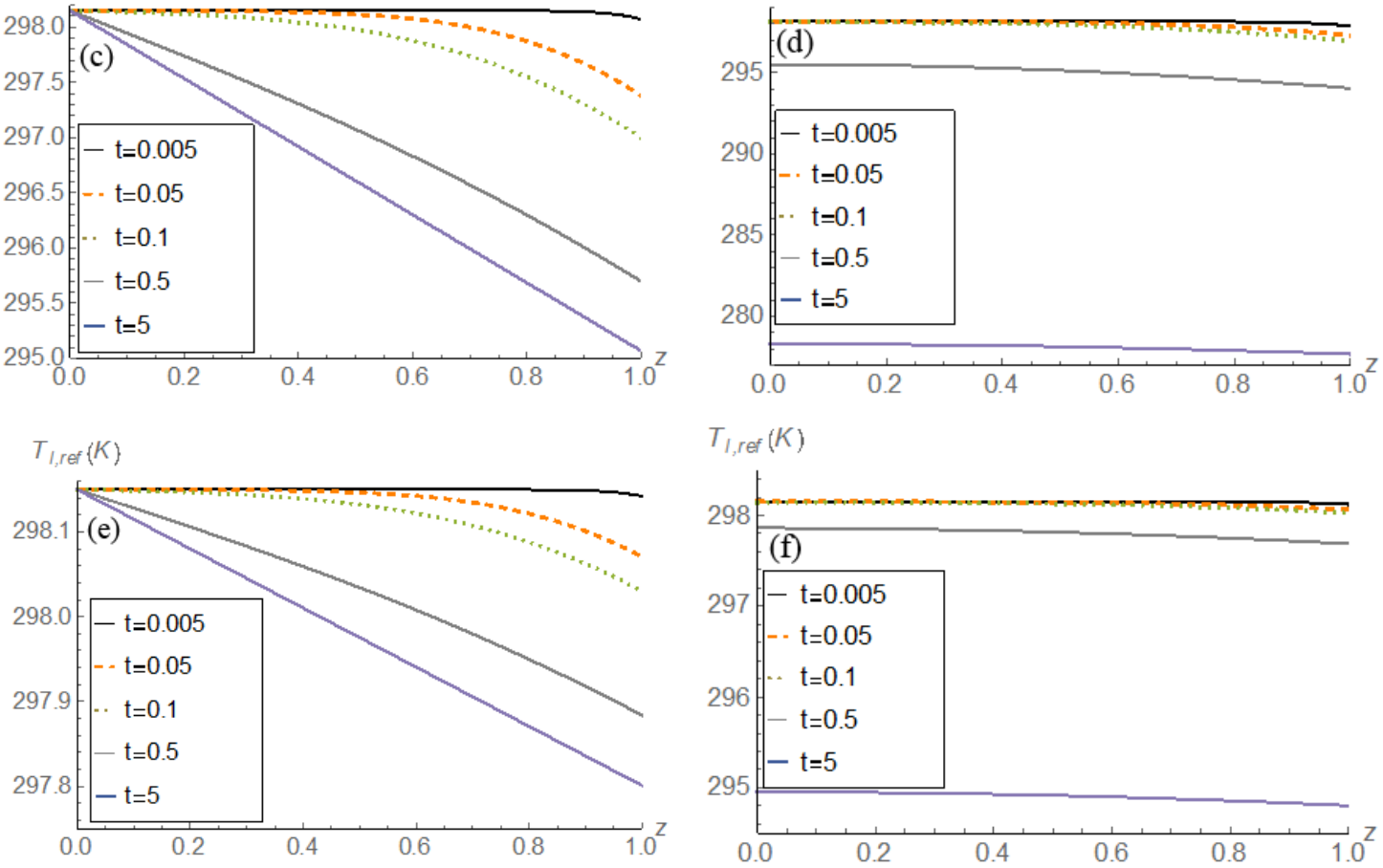

FIG. 2. (Color online) Temperature reference profiles in the liquid in the horizontal-layer case at different dimensionless times for $H=2$ ((a) and (b)), $H=11((\mathrm{c})$ and (d)) and $H=101$ ((e) and (f)) for the fixed-temperature ((a), (c) and (e)) and the zero-flux conditions at the bottom ((b), (d) and (f)).

From Fig. 2, we can see that as time passes a thermal boundary layer grows into the liquid layer and that a linear profile is eventually attained when considering the fixed-temperature condition at the bottom, with a slope $\left(T_{\Sigma, r e f}-T_{a m b}\right) / h_{l i q}$ (practically attained at $\left.t=5\right)$. The slope becomes smaller for higher $H$ values, which is easy to understand noting that the evaporation rate is smaller for higher $H$ values and thus the cooling at the interface is weaker. When considering the zero-flux condition at the bottom, the reference temperature profile tends to a spatially uniform one in the liquid. This constant value can be understood as the state in which the cooling due to evaporation is exactly compensated by the heat transfer from the warmer ambient atmosphere. Fig. 3 shows that the reference temperature profiles in the droplet exhibit qualitatively the same behavior as in the layer with the zero- 


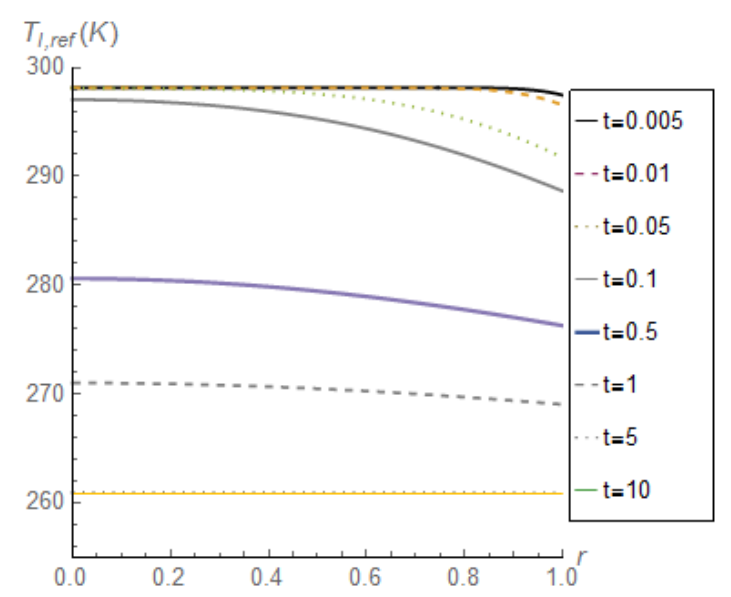

FIG. 3. (Color online) Temperature reference profiles in the liquid at different dimensionless times in the spherical-droplet case.

flux condition at the bottom, the temperature also tending to a constant value (which is attained at nearly the same time as for the case $H=2$ ), as expected.

\section{STABILITY ANALYSIS}

In this section, we provide the tools to perform the linear stability analysis (frozen-time approach). We start in subsection IV A by introducing the normal modes of perturbation. Then, using the hypotheses formulated in section II, we consider first the gas phase (subsection IV B) in order to subsequently arrive at an appropriate one-layer formulation in the liquid followed by the derivation of the instability criteria (subsection IV C).

\section{A. Description of perturbations}

For the horizontal layer, the linear perturbations upon the reference state for $w_{l}, T_{l}, \chi_{g}$, $T_{g}$ and $J$ (the only dependent variables appearing in the analysis that follows, where $w_{l}$ is the vertical component of the velocity field, noting also that actually $w_{l, r e f}=0$ ) are represented as

$$
e^{\sigma t+i\left(k_{x} x+k_{y} y\right)}\left\{W_{l}(z), \mathcal{T}_{l}(z), X_{g}(z), \mathcal{T}_{g}(z), \Phi\right\}
$$

respectively. Here, $\sigma$ is the (generally complex) growth rate of the perturbations, $\vec{k}=\left(k_{x}, k_{y}\right)$ is the wavevector (with the wavenumber $k \equiv \sqrt{k_{x}^{2}+k_{y}^{2}}$ ) and $x$ and $y$ are the horizontal Cartesian coordinates. The symbols in braces are the corresponding complex amplitudes (functions of $z$, except for $\Phi$ ). Note that in view of the symmetry, the complex amplitudes depend just on $k$, and not on $k_{x}$ and $k_{y}$ separately.

For the spherical droplet, the corresponding representation of the perturbations for $u_{r l}$, $T_{l}, \chi_{g}, T_{g}$ and $J\left(u_{r l}\right.$ being the radial component of the velocity field, $\left.u_{r l, r e f}=0\right)$ is 


$$
e^{\sigma t} Y_{l}^{m}(\theta, \phi)\left\{\frac{U_{l}(r)}{r}, \mathcal{T}_{l}(r), X_{g}(r), \mathcal{T}_{g}(r), \Phi\right\}
$$

respectively, the factor $1 / r$ being introduced in the first term in braces just for convenience. Here, $\sigma$ is again the (generally complex) growth rate of the perturbations and $\theta$ and $\phi$ are the polar and azimuthal angles of the spherical coordinate system. The spherical harmonics $Y_{l}^{m}(\theta, \phi)=P_{l}^{m}(\cos (\theta)) e^{i m \phi}$ satisfy the following equation:

$$
\mathcal{L}^{2} Y_{l}^{m}(\theta, \phi)=-l(l+1) Y_{l}^{m}(\theta, \phi)
$$

where

$$
\mathcal{L}^{2}=\frac{1}{\sin \theta} \frac{\partial}{\partial \theta}\left(\sin \theta \frac{\partial}{\partial \theta}\right)+\frac{1}{\sin ^{2} \theta} \frac{\partial^{2}}{\partial \phi^{2}}=r^{2} \nabla^{2}-\frac{\partial}{\partial r}\left(r^{2} \frac{\partial}{\partial r}\right)
$$

with $P_{l}^{m}(\cos \theta)$ being the associated Legendre polynomials. Here $l=0,1,2, \ldots$, and $m$ is an integer such that $-l \leq m \leq l$. In view of the symmetry, the complex amplitudes are functions of only $l$, and not of $m$. Note that $l$ is here the (discrete) counterpart of the wavenumber $k$.

It is worth mentioning that the formulae of the present subsection are identical in dimensional and dimensionless forms provided that the dimensionless $\sigma$ and $k$ are defined in terms of their dimensional counterparts as $\sigma \ell^{2} / \kappa_{l}$ and $k \ell$ (cf. the end of subsection III B).

\section{B. Perturbations in the gas phase and the Biot number for the perturbations}

In the present subsection, it is convenient to work in terms of dimensional variables. In accordance with the hypotheses outlined in section II, the perturbations of $\chi_{g}$ and $T_{g}$ satisfy just the Laplace equation. In terms of the complex amplitudes, for the horizontal-layer configuration, we have $\partial^{2} X_{g} / \partial z^{2}-k^{2} X_{g}=0$ and $\partial^{2} \mathcal{T}_{g} / \partial z^{2}-k^{2} \mathcal{T}_{g}=0$. The corresponding equations for the spherical-droplet configuration are $r^{2} \partial^{2} X_{g} / \partial r^{2}+2 r \partial X_{g} / \partial r-l(l+1) X_{g}=0$ and $r^{2} \partial^{2} \mathcal{T}_{g} / \partial r^{2}+2 r \partial \mathcal{T}_{g} / \partial r-l(l+1) \mathcal{T}_{g}=0$. The perturbations vanish $\left(X_{g}=0\right.$ and $\left.\mathcal{T}_{g}=0\right)$ where the ambient conditions are supposed to be maintained, i.e. at the top fictive boundary of the gas layer $\left(z=h_{\text {liq }}+h_{\text {gas }}\right)$ in the first configuration and far away from the droplet $(r \rightarrow \infty)$ in the second configuration. The solutions can then be written as

$$
X_{g}=\left\{\begin{array}{c}
X_{g \Sigma} \frac{\sinh \left[k\left(h_{l i q}+h_{g a s}-z\right)\right]}{\sinh \left[k h_{g a s}\right]} \text { (layer) } \\
X_{g \Sigma} \frac{R^{l+1}}{r^{l+1}} \text { (droplet) }
\end{array}\right.
$$

and 


$$
\mathcal{T}_{g}=\left\{\begin{array}{c}
\mathcal{T}_{\Sigma} \frac{\sinh \left[k\left(h_{\text {liq }}+h_{\text {gas }}-z\right)\right]}{\sinh \left[k h_{\text {gas }}\right]} \text { (layer) } \\
\mathcal{T}_{\Sigma} \frac{R^{l+1}}{r^{l+1}} \quad \text { (droplet) }
\end{array}\right.
$$

where the subscript ' $\Sigma$ ' once again refers to the values at the interface $\left(z=h_{\text {liq }}\right.$ for the layer and $r=R$ for the droplet).

The quantities $\chi_{g \Sigma}$ and $T_{\Sigma}$ are related by the Clausius-Clapeyron relation

$$
\chi_{g \Sigma}=\frac{p_{s a t}\left(T_{a m b}\right)}{p_{a m b}} e^{-\frac{L M}{R g}\left(\frac{1}{T_{\Sigma}}-\frac{1}{T_{a m b}}\right)} \approx \frac{p_{s a t}\left(T_{a m b}\right)}{p_{a m b}} e^{\frac{L M\left(T_{\Sigma}-T_{a m b}\right)}{R_{g} T_{a m b}^{2}}}
$$

where it is taken into account that the difference between the absolute temperatures $T_{\Sigma}$ and $T_{a m b}$ is not expected to be large ("Frank-Kamenetskii transformation"), which is consistent with neglecting the temperature dependence of the material properties of the fluids. Linearizing and thus adapting (26) to the perturbations, we obtain

$$
X_{g \Sigma}=\frac{L M}{R_{g} T_{a m b}^{2}} \chi_{g \Sigma, r e f} \mathcal{T}_{\Sigma}
$$

Similarly, adapting Eq. (10) to the perturbations, albeit without perturbing $\left(1-\chi_{g \Sigma}\right)$ in the denominator (see [28]), and using Eq. (24) yields

$$
\Phi=\left\{\begin{array}{c}
\frac{p_{a m b} D_{g} M}{R_{g} T_{a m b}} \frac{1}{1-\chi_{g \Sigma, r e f}} k \operatorname{coth}\left[k h_{g a s}\right] X_{g \Sigma} \quad \text { (layer) } \\
\frac{p_{a m b} D_{g} M}{R_{g} T_{a m b}} \frac{1}{1-\chi_{g \Sigma, r e f}} \frac{l+1}{R} X_{g \Sigma} \text { (droplet) }
\end{array}\right.
$$

Further analysis of perturbations must involve the liquid phase, which is accomplished in the next subsection. However, an important intermediate step in this direction and at the same time a final touch to the gas-phase consideration can be made by just invoking the interface energy balance (cf. Eq. (14) and the text below it), which for the perturbations writes as

$$
-\lambda_{l} \frac{\partial \mathcal{T}_{l}}{\partial \mathbf{n}}+\lambda_{g} \frac{\partial \mathcal{T}_{g}}{\partial \mathbf{n}}=\Phi L
$$

Using Eqs. (25), (27) and (28) in Eq. (29) brings to an interface condition in the form

$$
\frac{\partial \mathcal{T}_{l}}{\partial \mathbf{n}}+\frac{B i}{\ell} \mathcal{T}_{l}=0
$$

with 


$$
B i=\left\{\begin{array}{c}
B i_{0} k h_{\text {gas }} \operatorname{coth}\left[k h_{\text {gas }}\right] \quad \text { (layer) } \\
B i_{0}(l+1) \quad \text { (droplet) }
\end{array}\right.
$$

being the Biot number for perturbations, where

$$
B i_{0}=\frac{1}{\delta_{g}}\left\{\frac{\lambda_{g}}{\lambda_{l}}+\frac{p_{a m b} L^{2} M^{2} D_{g}}{R_{g}^{2} T_{a m b}^{3} \lambda_{l}} \frac{\chi_{g \Sigma, r e f}}{1-\chi_{g \Sigma, r e f}}\right\}
$$

is the value of $B i$ at $k=0 / l=0$ (uniform perturbation). Recall here also Eq. (7). Note the dependence of $B i$ on the wavenumber ( $B i$ is not merely equal to $B i_{0}$ as in [18], [19], if we put it in our terms), showing the importance of which is the principal goal of the present paper. Note also that the distinction between $B i_{0}$ and $B i_{0, a m b}$, earlier defined in Eq. (20), is just that the latter is a version of the former evaluated at the ambient conditions. On account of Eq. (4), this can symbolically be written as

$$
B i_{0, a m b}=\left.B i_{0}\right|_{T_{\Sigma, r e f} \equiv T_{a m b}}
$$

Should the linearization (18) have been adopted, we would here arrive at the same expression (31), with a manifest $k$-dependence, but just with $B i_{0}$ replaced by $B i_{0, a m b}$. This would still contrast with the approach used elsewhere [17], [29], corresponding in our terms to $B i \equiv B i_{0, a m b}$, even for the perturbations.

\section{Perturbations in the liquid phase}

In the present subsection, we shall work in dimensionless variables (see the ends of subsections III B and IV A). Besides, the velocity is made dimensionless with the scale $\kappa_{l} / \ell$.

Furthermore, we shall limit ourselves to looking for marginal-stability conditions (monotonic instability), and hence set

$$
\sigma=0
$$

First, we turn to the consideration of the velocity field, which is crucial here unlike the situation in the gas layer. In the context of the linear stability analysis in both configurations under consideration, the problem can actually be formulated in terms of the normal velocity field component only (e.g. [4], [18], see also Appendix A). In particular, from the linearized Navier-Stokes equations in terms of the normal-mode amplitudes, one obtains (for $\sigma=0$ )

$$
\begin{gathered}
\left(D_{z}^{2}-k^{2}\right)^{2} W_{l}=0 \quad \text { (layer) } \\
\left(D_{r}^{2}+\frac{2}{r} D_{r}-\frac{l(l+1)}{r^{2}}\right)^{2} U_{l}=0 \quad \text { (droplet) }
\end{gathered}
$$

where $D_{z} \equiv d / d z$ and $D_{r} \equiv d / d r$.

As the interface is assumed underformable and the evaporation-induced velocity in the liquid is much smaller than $\kappa_{l} / \ell$ and the Marangoni-induced velocity (see section II), the interface boundary conditions include $W_{l}=0$ at $z=1$ and $U_{l}=0$ at $r=1$. The impermeability and no-slip conditions at the bottom of the liquid layer are $W_{l}=0=D_{z} W_{l}$ at 
$z=0$, and the no-singularity condition in the center of the droplet is $U_{l}=0$ at $r=0$. The solution of (33) satisfying these boundary conditions is

$$
\begin{gathered}
W_{l}=A W_{l}^{*} \text { (layer) } \\
U_{l}=A U_{l}^{*} \text { (droplet) }
\end{gathered}
$$

where $A$ is a free coefficient and

$$
\begin{gathered}
W_{l}^{*}=k z \cosh (k z)-(1-z+k z \operatorname{coth}(k)) \sinh (k z) \text { (layer) } \\
U_{l}^{*}=r^{l}-r^{l+2} \text { (droplet) }
\end{gathered}
$$

The tangential-stress balance at the interface, including the Marangoni (thermocapillary) stresses, can be expressed in terms of the normal velocity only as well (e.g. [4], [18], see also Appendix B):

$$
\begin{gathered}
D_{z}^{2} W_{l}+M a k^{2} \mathcal{T}_{l}=0 \text { at } z=1 \text { (layer) } \\
D_{r}^{2} U_{l}+\operatorname{Mal}(l+1) \mathcal{T}_{l}=0 \text { at } r=1 \text { (droplet) }
\end{gathered}
$$

where

$$
M a=\frac{\gamma_{T} \ell \theta_{T}}{\kappa_{l} \mu_{l}}
$$

is the (thermal) Marangoni number, $\gamma_{T} \equiv-d \gamma / d T$ ( $>0$ for most fluids, including our HFE) and $\gamma$ is the surface tension. Recall that the contribution from the gas side is neglected in (36) in view of a small gas viscosity (cf. section II). Note that $M a>0$ here.

Next, we turn to the heat-transfer equation $\partial T_{l} / \partial t+\vec{v}_{l} \cdot \nabla T_{l}=\nabla^{2} T_{l}$, which is linearized about the reference state and expressed in terms of the amplitudes to yield (for $\sigma=0$ )

$$
\begin{gathered}
\left.W_{l} D_{z} T_{l, \text { ref }}=\left(D_{z}^{2}-k^{2}\right) \mathcal{T}_{l} \quad \text { (layer }\right) \\
\frac{U_{l}}{r} D_{r} T_{l, \text { ref }}=\left(D_{r}^{2}+\frac{2}{r} D_{r}-\frac{l(l+1)}{r^{2}}\right) \mathcal{T}_{l} \quad \text { (droplet) }
\end{gathered}
$$

The effective interface heat-transfer condition (30) with (31) is now rendered in dimensionless form, becoming

$$
\begin{gathered}
\frac{\partial \mathcal{T}_{l}}{\partial \mathbf{n}}+B i \mathcal{T}_{l}=0 \text { at } z=1 / r=1 \\
B i=\left\{\begin{array}{r}
B i_{0} k(H-1) \operatorname{coth}[k(H-1)] \text { (layer) } \\
B i_{0}(l+1) \text { (droplet) }
\end{array}\right.
\end{gathered}
$$

where $B i_{0}$ is still defined by (32). 
At the other end of the liquid domain, i.e. at the bottom of the layer and in the center of the droplet, we have the following conditions for the temperature:

$$
\begin{gathered}
\mathcal{T}_{l}=0 \text { at } z=0 \text { (layer FTB) } \\
D_{z} \mathcal{T}_{l}=0 \text { at } z=0 \text { (layer TIB) } \\
\mathcal{T}_{l}<\infty \text { at } r=0 \text { (droplet) }
\end{gathered}
$$

where FTB stands for the case of a "fixed temperature at the bottom" while TIB for the case of a "thermally insulated bottom".

The solution of problem (38) with (34) for $\mathcal{T}_{l}$ can be expressed as

$$
\begin{gathered}
\mathcal{T}_{l}=\frac{e^{k z}}{2 k} A\left(C_{1}-\int_{z}^{1} e^{-k z^{\prime}} W_{l}^{*}\left(k, z^{\prime}\right) \frac{\partial T_{l, r e f}\left(z^{\prime}, t\right)}{\partial z^{\prime}} d z^{\prime}\right) \\
+\frac{e^{-k z}}{2 k} A\left(C_{2}+\int_{z}^{1} e^{k z^{\prime}} W_{l}^{*}\left(k, z^{\prime}\right) \frac{\partial T_{l, r e f}\left(z^{\prime}, t\right)}{\partial z^{\prime}} d z^{\prime}\right) \quad \text { (layer) } \\
\mathcal{T}_{l}=\frac{r^{l}}{2 l+1} A\left(C_{1}-\int_{r}^{1} r^{\prime-l} U_{l}^{*}\left(l, r^{\prime}\right) \frac{\partial T_{l, r e f}\left(r^{\prime}, t\right)}{\partial r^{\prime}} d r^{\prime}\right) \\
+\frac{r^{-(l+1)}}{2 l+1} A\left(C_{2}+\int_{r}^{1} r^{l+1} U_{l}^{*}\left(l, r^{\prime}\right) \frac{\partial T_{l, r e f}\left(r^{\prime}, t\right)}{\partial r^{\prime}} d r^{\prime}\right) \quad \text { (droplet) }
\end{gathered}
$$

where $W_{l}^{*}=W_{l}^{*}(k, z)$ and $U_{l}^{*}=U_{l}^{*}(l, r)$ are given by $(35)$, while $T_{l, r e f}=T_{l, r e f}(z, t)$ (layer) and $T_{l, \text { ref }}=T_{l, \text { ref }}(r, t)$ (droplet) are the dimensionless (transient) reference temperature profiles determined in section III. The integration constants $C_{1}$ and $C_{2}$ can be found using the boundary conditions (39) and (41).

Finally, the tangential-stress balance (36) serves as the compatibility condition in the present scheme. Substituting (34) and (42) with the determined values of $C_{1}$ and $C_{2}$ in there, $A$ cancels out, and we obtain the values of the Marangoni number for which there exists a neutral monotonic perturbation $(\sigma=0)$

$$
\begin{gathered}
M a=\frac{2(k \operatorname{coth}(k)+B i)(\sinh (k) \cosh (k)-k)}{k \int_{0}^{1} \sinh (k z) W_{l}^{*}(k, z) \frac{\partial T_{l, r e f}(z, t)}{\partial z} d z} \text { (layer FTB) } \\
M a=\frac{2(k+B i \operatorname{coth}(k))(\sinh (k) \cosh (k)-k)}{k \int_{0}^{1} \cosh (k z) W_{l}^{*}(k, z) \frac{\partial T_{l, r e f}(z, t)}{\partial z} d z} \text { (layer TIB) } \\
M a=\frac{2(B i+l)(2 l+1)}{l(l+1) \int_{0}^{1}\left(r^{2 l+3}-r^{2 l+1}\right) \frac{\partial T_{l, r e f}(r, t)}{\partial r} d r}
\end{gathered}
$$

where recall that $W_{l}^{*}$ is given by (35), while $T_{l, r e f}$ was numerically determined in section III. In this way, we obtain $M a$ as functions of the wavenumber ( $k$ for the layer, and $l$ for the droplet) representing the marginal condition ("marginal curve"), i.e. the boundary in the parameter space between the decaying and growing perturbations. In all the cases considered in the present paper, it turns out that the marginal curve corresponds to $M a>0$, which is in agreement with the sign of $M a$ in (37). The critical Marangoni number for the instability onset is then obtained by minimizing $M a$ at the marginal condition with respect to $k$ or $l$. Here, recall that $0<k<\infty$, whereas $l$ has discrete values $l=1,2, \ldots$. As the reference temperature profiles are here generally transient (functions of $t$ ), while the frozentime approach is adopted for the linear stability analysis, the critical Marangoni numbers hereby obtained are also function of $t$. On account of (37), for each concrete liquid case (e.g. 
HFE-7100), the results can equivalently be presented in terms of the critical values of $\ell$, i.e. of the layer thickness $h_{l i q}$ or of the droplet radius $R$, which is what we shall do in section $\mathrm{V}$. Finally, remember that the Biot number $B i$ appearing in the results (43) is wavenumberdependent as given by Eq. (40) with Eq. (32). In section V, we shall see how this affects the results as compared to a simplified treatment with just $B i \equiv B i_{0}$ independent of the wavenumber.

\section{RESULTS}

\section{A. Stability of transient reference states}

The results for the critical size of the system (thickness $h_{l i q}$ for the layer, and radius $R$ of the droplet) as a function of (both dimensional and dimensionless) time are represented in Fig. 4. The cases of a layer with a fixed temperature at the bottom (FTB), of a layer with an insulated bottom (TIB) and of a droplet are shown.

The computation has been carried out for the HFE-7100 liquid with the properties shown in Table I. The time is actually represented along the ordinate axis, and the results can also be interpreted inversely, as the critical time for the instability onset as a function of the system size. The results with the dimensionless time are also shown in order to allow connecting the temperature profiles from Figs. 2 and 3 to the results in Fig. 4. As announced at the end of section IV, the results are shown not only based upon the ultimate, wavenumber-dependent expression (40) for the Biot number but also, for comparison, for $B i \equiv B i_{0}$, the latter mimicking the typical arrangement used in the literature. To appreciate the values of the Biot number encountered here, let us mention that $B i_{0, a m b} \approx 1.697 / \delta_{g}$ for the parameters of Table I ( $B i_{0}$ being slightly different and dependent on the configuration and indirectly on time). In the layer case, the problem also depends on an additional parameter, the ratio of the gas and liquid thicknesses $H$, and the study is carried out at fixed $H$ values, the results being shown for $H=2,11$ and 101. The states with growing perturbations present within the frozen-time analysis are located on the concave side of the curve in each case, the corresponding region indicated by "unstable" in Fig. 4.

We observe from Fig. 4 that in each case there is a minimum liquid thickness, below which no growing perturbations occur at any time. In the case of a fixed temperature at the bottom of the layer, this minimum liquid thickness is actually associated with a vertical asymptote corresponding to the stationary reference state with a constant temperature gradient eventually attained after the transients, as $t \rightarrow \infty$ (see also subsection V B). In the case of a layer with an insulated bottom, as well as in the case of a droplet, there is no such vertical asymptote, and the system regains its stability at sufficiently large $t$ values. This is not surprising given that in these two cases the system eventually tends to a reference state with a uniform temperature in the liquid, the heat needed for evaporation being supplied by thermal conduction through the gas phase. The minimum liquid thickness for the latter situation is referred to as a turning point [23] and the influence of the bottom boundary condition on the appearance of such a turning point is discussed in [24] for a binary liquid in the solutal Marangoni case. Note that the critical wavenumbers for the layer-case are continuous, which makes it difficult to present them on the plots in Fig. 4. Therefore, separate plots have been made in Fig. 5, representing the (dimensionless) critical wavenumbers as a function of the liquid thickness, corresponding to the FTB and TIB cases in Fig. 4. The wavenumbers are represented by markers connected with each other by a line. The 

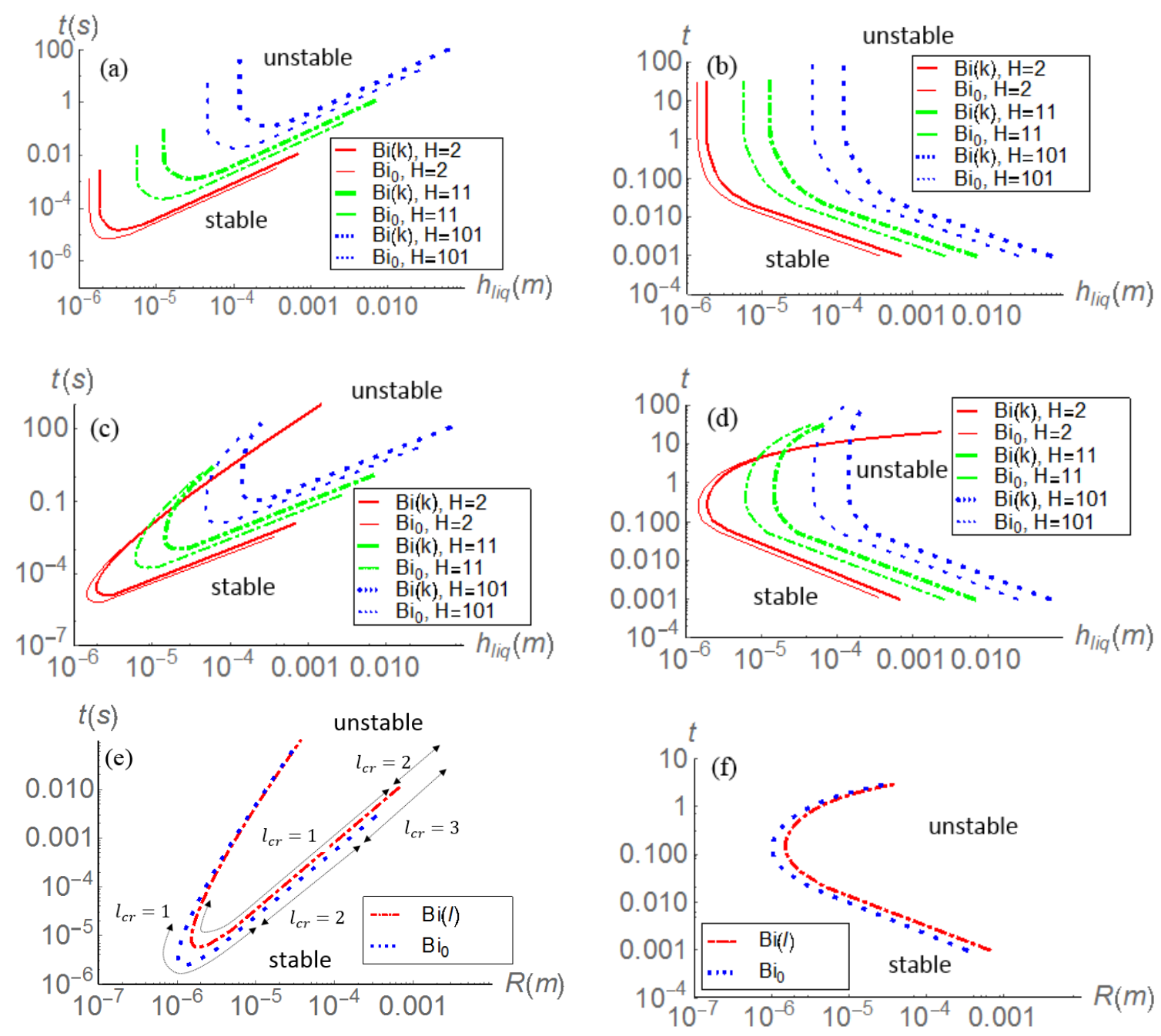

FIG. 4. (Color online) Dimensional ((a), (c) and (e)) and dimensionless ((b), (d) and (f)) time versus the system size at the critical condition for a layer with FTB ((a) and (b)), a layer with TIB ((c) and (d)) (both for $H=2,11$ and 101), and a droplet ((e) and (f)), comparing the results using the wavenumber-dependent Biot number with those using just a constant Biot number $B i_{0}$ as typically the case in the literature (e.g. [15],[18],[29]).

critical wavenumbers for the droplet-case are, however, discrete and shown for appreciation in Fig. 4.

Now, as far as the principal objective of the present paper is concerned, namely the importance of considering a wavenumber-dependent $B i$ instead of just $B i \equiv B i_{0}$, we observe quite an appreciable effect in the results of Fig. 4. The effect is especially pronounced for large values of $H$ in the layer case. Indeed, the $k$-dependence in $B i$ accounts for the diffusional exchange through the gas phase between spots at different temperatures on the liquid-gas interface, whereas $B i_{0}$ measures such a diffusional exchange between the interface and the ambient atmosphere. For large $H$ (large relative gas-layer thicknesses), the first exchange clearly tends to dominate over the second one, hence a strong effect upon the results. On the contrary, for moderate $H$, the effect is less pronounced, and so it is in the case of a droplet, qualitatively similar to $H=2$ for the layer. Note though a significant role 

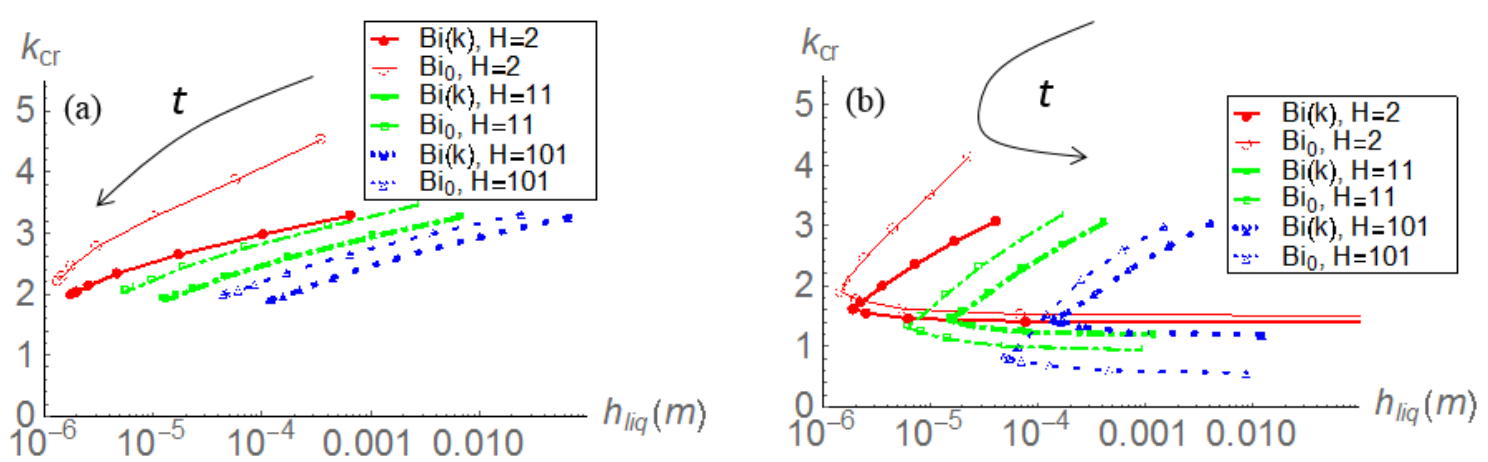

FIG. 5. (Color online) Critical wavenumbers versus the layer thickness in the FTB (a) and TIB (b) cases (both for $H=2,11$ and 101), comparing the results using the wavenumber-dependent Biot number with those using just a constant Biot number $B i_{0}$. The direction of time along the curves is symbolically indicated by the $t$ arrows

of the studied effect on the critical wavenumber selection in the droplet case (Fig. 4). Note also that if the gas properties were not treated in a simplified way as we did in the present paper (negligible dynamic viscosity and unconditionally large diffusivity), it could be found that the excitation of the mode $l=1$ is actually accompanied by translational motion of the droplet [31].

\section{B. Pearson's marginal curves in the layer FTB case}

It is also instructive to illustrate the role of the wavenumber dependency of the Biot number in terms of the marginal condition as a whole, i.e. the marginal curve of the Marangoni number as a function of the wavenumber, and not just in terms of the critical condition (the minimum of this curve) as done in subsection V A. In doing so, we shall here limit ourselves to the case of a layer with a fixed temperature at the bottom (FTB), and even more specifically to the final steady reference state with a constant temperature gradient across the layer (in particular, corresponding to the vertical asymptotes in the upper plot of Fig. 4). Using

$$
\partial T_{l, r e f} / \partial z=-\delta_{\theta}=\text { const }
$$

together with Eq. (35) in Eq. (43) for the layer FTB case, we arrive at the classical Pearson marginal curve (cf. e.g. [1],[4],[21])

$$
M a=\delta_{\theta}^{-1} 8 k \frac{(k \cosh (k)+B i \sinh (k))(\sinh (k) \cosh (k)-k)}{\sinh ^{3}(k)-k^{3} \cosh (k)} \quad \text { (layer FTB) }
$$

the form of which does not depend on whether $B i$ is $k$-dependent or not. Note that $\delta_{\theta} \neq 1$ merely due to the fact that the Marangoni number is here defined using a temperature scale $\theta_{T}$ not coinciding with the actual temperature difference across the liquid layer in the reference state (cf. Eq. (37) and the end of subsction III B). We have $\delta_{\theta}=\left(T_{a m b}-T_{\Sigma, r e f}\right) / \theta_{T}$. $T_{\Sigma, r e f}$ is here dimensional and determined according to the procedure described in section III. However, as in the present subsection we deal with the layer FTB case and a steady reference 
state thereof with a constant temperature gradient, this task is simplified. In particular, Eqs. (11) and (14) can just be reduced to

$$
\left(\lambda_{l}+\frac{\lambda_{g}}{H-1}\right)\left(T_{a m b}-T_{\Sigma, r e f}\right)=L \frac{p_{a m b} D_{g} M}{R_{g} T_{a m b}} \frac{\chi_{g \Sigma, r e f}-\chi_{a m b}}{1-\chi_{g \Sigma, r e f}} \frac{1}{H-1}
$$

and thus $T_{\Sigma, r e f}$ is here calculated from the system of equations given by (4) and (45).

The results are shown in Fig. 6 for the same cases as in Fig. 4 for the FTB-layer-case.

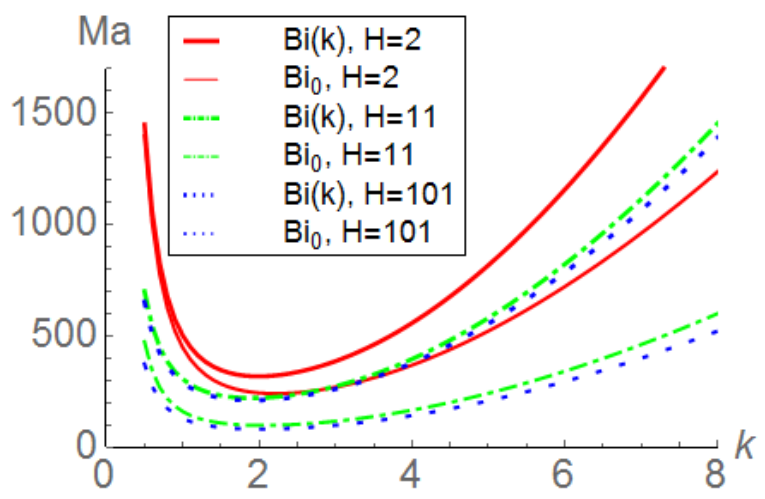

FIG. 6. (Color online) The Pearson-like marginal curves for steady reference states (linear temperature profiles) of the layer with FTB. The same arrangements as in Fig. 4 are explored.

Here, one can once again appreciate the importance of considering a $k$-dependent Biot number. However, now we can observe this not only for the critical values (the minima of the curves), but also for the width of the instability interval at finite supercriticalities. This points to the importance of the phenomenon for nonlinear regimes of Marangoni convection too.

\section{CONCLUSIONS}

A linear stability analysis has here been carried out for the Marangoni (thermocapillary) instability in two different evaporative systems: a horizontal layer and a spherical droplet of a pure liquid, both evaporating into ambient air. For the layer, two subcases have been considered: a bottom with a fixed temperature (equal to the ambient one) and a thermally insulated bottom. As a concrete example, the HFE-7100 liquid has been used. Transient reference states evolving from an initially uniform temperature everywhere (equal to the ambient temperature) have been considered.

The goal of the paper was to put into evidence the intricacies of the definition of the effective transfer coefficients (the Biot numbers) at the liquid-gas interface arising in the framework of one-sided formulations and representing the combined effect of evaporation and heat transfer through the gas phase. Namely, it has been shown that the Biot numbers suitable for the description of the horizontally/spherically uniform reference states may no longer be suitable for perturbations, for which the appropriately defined Biot numbers must in fact be wavenumber-dependent. Physically, such a wavenumber dependence comes from the interaction between different regions of the interface, and not just between the 
interface and the ambient medium as for the "uniform" Biot numbers. Eqs. (31) or (40) provide an example of how a uniform Biot number can be generalized into a corresponding wavenumber-dependent one, which can also be considered as a possible practical recipe for such a generalization when one proceeds from homogeneous-state transfer coefficients.

While the scope of the linear stability analysis chosen for illustrative purposes in the present paper is limited to monotonic instability thresholds and a frozen-time approach, the results nonetheless indicate that the wavenumber-dependent Biot numbers must be equally important both in the more general context of linear stability theories and in the framework of a nonlinear description of the ensuing convection.

\section{ACKNOWLEDGEMENTS}

The authors gratefully acknowledge the financial support of the European Space Agency and of the Belgian Science Policy through the PRODEX projects. P.C. also acknowledges the financial support of the Fonds de la Recherche Scientifique (FNRS).

\section{APPENDICES}

\section{A. Equations for $W_{l}$ and $U_{l}$}

The linearized dimensionless vector-form Navier-Stokes equations in the liquid can be written as

$$
\begin{array}{r}
\vec{\nabla} \cdot \vec{v}_{l}=0, \\
P r_{l}^{-1} \frac{\partial \vec{v}_{l}}{\partial t}=-\vec{\nabla} p_{l}+\nabla^{2} \vec{v}_{l}
\end{array}
$$

where $\vec{v}_{l}$ is the velocity field, $p_{l}$ the pressure field, $P r_{l}=\nu_{l} / \kappa_{l}$ the Prandtl number, $\nu_{l}$ the kinematic viscosity, and $\kappa_{l}$ the thermal diffusivity. Applying $\vec{\nabla} \times \vec{\nabla} \times$ to the momentum equation, one obtains

$$
\operatorname{Pr}_{l}^{-1} \frac{\partial}{\partial t} \nabla^{2} \vec{v}_{l}=\nabla^{2} \nabla^{2} \vec{v}_{l}
$$

on account of the identity $\vec{\nabla} \times \vec{\nabla} \times \vec{Q}=-\nabla^{2} \vec{Q}$, valid for a solenoidal vector field $\vec{Q}$ (with $\vec{\nabla} \cdot \vec{Q}=0)$, such as $\vec{v}_{l}$.

In the layer case, it is straightforward to identify the vertical component of Eq. (47):

$$
\operatorname{Pr}_{l}^{-1} \frac{\partial}{\partial t} \nabla^{2} w_{l}=\nabla^{2} \nabla^{2} w_{l}
$$

With the normal modes (21), this yields

$$
-\operatorname{Pr}_{l}^{-1} \sigma\left(D_{z}^{2}-k^{2}\right) W_{l}+\left(D_{z}^{2}-k^{2}\right)^{2} W_{l}=0
$$


which for $\sigma=0$ results in Eq. (33) for the layer.

In the spherical droplet case, we project Eq. (47) on the position vector $\vec{r}$ drawn from the center of the droplet. Using the identity $\vec{r} \cdot \nabla^{2} \vec{Q}=\nabla^{2}(\vec{r} \cdot \vec{Q})$ valid for a solenoidal vector field $\vec{Q}$, and taking into account that both $\nabla^{2} \vec{v}_{l}$ and $\vec{v}_{l}$ are solenoidal and the fact that $\vec{r} \cdot \vec{v}_{l}=r u_{r l}$, we arrive at

$$
\operatorname{Pr}_{l}^{-1} \frac{\partial}{\partial t} \nabla^{2}\left(r u_{r l}\right)=\nabla^{2} \nabla^{2}\left(r u_{r l}\right)
$$

With the normal modes (22) (see also Eq. (23)), this yields

$$
-P r_{l}^{-1} \sigma\left(D_{r}^{2}+\frac{2}{r} D_{r}-\frac{l(l+1)}{r^{2}}\right) U_{l}+\left(D_{r}^{2}+\frac{2}{r} D_{r}-\frac{l(l+1)}{r^{2}}\right)^{2} U_{l}=0
$$

which with $\sigma=0$ becomes Eq. (33) for the droplet.

\section{B. Deduction of the Marangoni condition}

Neglecting the air viscosity, the dimensionless tangential stress balance at the interface can be written in the layer case as

$$
\begin{aligned}
& \left(\frac{\partial w_{l}}{\partial x}+\frac{\partial u_{l}}{\partial z}\right)+M a \frac{\partial T_{l}}{\partial x}=0 \\
& \left(\frac{\partial w_{l}}{\partial y}+\frac{\partial v_{l}}{\partial z}\right)+M a \frac{\partial T_{l}}{\partial y}=0
\end{aligned}
$$

We can now apply $\partial_{x}$ and $\partial_{y}$ to Eqs. (52) and (53), respectively, sum the results up and apply the continuity equation $\partial_{x} u+\partial_{y} v+\partial_{z} w=0$ in order to find

$$
\left(\frac{\partial^{2} w_{l}}{\partial x^{2}}+\frac{\partial^{2} w_{l}}{\partial y^{2}}-\frac{\partial^{2} w_{l}}{\partial z^{2}}\right)+M a\left(\frac{\partial^{2} T_{l}}{\partial x^{2}}+\frac{\partial^{2} T_{l}}{\partial y^{2}}\right)=0
$$

Using Eq. (21) in Eq. (54) gives

$$
\left(D_{z}^{2}+k^{2}\right) W_{l}+k^{2} M a \mathcal{T}_{l}=0
$$

With $W_{l}=0$ at the interface (as explained in the main text), we recover Eq. (36) for the layer.

For the droplet, a similar procedure is performed. The conditions expressing the dimensionless tangential stress balance at $r=1$ in the spherical coordinates are 


$$
\begin{gathered}
\left(r \frac{\partial}{\partial r}\left(\frac{u_{\theta l}}{r}\right)+\frac{1}{r} \frac{\partial u_{r l}}{\partial \theta}\right)+\frac{M a}{r} \frac{\partial T_{l}}{\partial \theta}=0 \\
\left(r \frac{\partial}{\partial r}\left(\frac{u_{\phi l}}{r}\right)+\frac{1}{r \sin \theta} \frac{\partial u_{r l}}{\partial \phi}\right)+\frac{M a}{r \sin \theta} \frac{\partial T_{l}}{\partial \phi}=0
\end{gathered}
$$

We can now apply $\frac{1}{\sin \theta} \partial_{\theta} \sin \theta$ and $\frac{1}{\sin \theta} \partial_{\phi}$ to Eqs. (56) and (57), respectively, sum the results up and apply the continuity equation

$$
\frac{1}{r^{2}} \frac{\partial}{\partial r}\left(r^{2} u_{r l}\right)+\frac{1}{r \sin \theta} \frac{\partial}{\partial \theta}\left(\sin \theta u_{\theta l}\right)+\frac{1}{r \sin \theta} \frac{\partial}{\partial \phi} u_{\phi l}=0
$$

in order to find

$$
\left(\frac{\partial^{2}}{\partial r^{2}}\left(r u_{r l}\right)-\frac{\left(2+\mathcal{L}^{2}\right)\left(r u_{r l}\right)}{r^{2}}\right)-\frac{M a}{r} \mathcal{L}^{2} T_{l}=0
$$

keeping in mind the definition of $\mathcal{L}^{2}$ following (23). Using the normal modes Eq. (22) in Eq. (58) yields

$$
\left(D_{r}^{2}-\frac{2-l(l+1)}{r^{2}}\right) U_{l}+M a \frac{l(l+1)}{r} \mathcal{T}_{l}=0
$$

With $U_{l}=0$ at the interface as before, Eq. (36) for the droplet is finally recovered.

[1] J.R.A. Pearson, "On convection cells induced by surface tension," J. Fluid Mech. 4, 489 (1958).

[2] H. Bénard, "Les tourbillons cellulaires dans une nappe liquide transportant de la chaleur en régime permanent," Ann. Chem. Phys. 23, 62 (1901).

[3] C. Normand, Y. Pomeau, M. Velarde, "Convective instability: a physicist's approach," Rev. Mod. Phys. 49, 581 (1977).

[4] P. Colinet, J.C. Legros, M.G. Velarde, Nonlinear Dynamics of Surface Tension Driven Instabilities (Wiley-VCH Verlag GmbH, Berlin, 2001).

[5] B. Haut, P. Colinet, "Surface-tension-driven instabilities of a pure liquid layer evaporating into an inert gas," J. Colloid Interface Sci. 285, 296 (2005).

[6] J. Margerit, M. Dondlinger, P.C. Dauby, "Improved 1.5-sided model for the weakly nonlinear study of Bénard-Marangoni instabilities in an evaporating liquid layer," J. Colloid Interface Sci. 290, 220 (2005).

[7] H. Mancini, D. Maza, Pattern formation without heating in an evaporative convection experiment," Europhys. Lett. 66, 812 (2004).

[8] B. Scheid, J. Margerit, C.S. Iorio, L. Joannes, M. Heraud, P. Queeckers, P.C. Dauby, P. Colinet, "Onset of thermal ripples at the interface of an evaporating liquid under a flow of inert gas," Exp. Fluids 52, 1107 (2012). 
[9] D. Merkt, M. Bestehorn, "Bénard-Marangoni convection in a strongly evaporating fluid," Physica D 185, 196 (2003).

[10] H. Liu, A.J. Valocchi, Y. Zhang, Q. Kang, "Phase-field-based lattice Boltzmann finitedifference model for simulating thermocapillary flows," Phys. Rev. E 87, 013010 (2013).

[11] S.G. Yiantsios, B.G. Higgins, "A mechanism of Marangoni instability in evaporating thin liquid films due to soluble surfactant," Phys. Fluids 22, 022102 (2010).

[12] F. Chauvet, S. Dehaeck, P. Colinet, Threshold of Bénard-Marangoni instability in drying liquid films," Europhys. Lett. 99, 34001 (2012).

[13] M. Bestehorn, "Convection in thick and in thin fluid layers with a free surface The influence of evaporation," Eur. Phys. J. S. T. 146, 391 (2007).

[14] R. Liu, Q. Liu, "Linear stability analysis of convection in two-layer system with an evaporating vapor-liquid interface," Acta Mech. Sinica 22, 109 (2006).

[15] Z.F. Sun, "Onset of Rayleigh-Bénard-Marangoni convection with time-dependent nonlinear concentration profiles," Chem. Eng. Science 68, 579 (2012).

[16] S.K. Wilson, "The onset of steady Marangoni convection in a spherical geometry," J. Engineering Math. 28, 427 (1994).

[17] F. Doumenc, T. Boeck, B. Guerrier, and M. Rossi, "Transient Rayleigh-Bénard-Marangoni convection due to evaporation: A linear non-normal stability analysis," J. Fluid Mech. 648, $521(2010)$.

[18] V.-M. Ha, C.-L. Lai, "The onset of stationary Marangoni instability of an evaporating droplet," Proc. Royal Soc. A 457, 885 (2001).

[19] V.-M. Ha, C.-L. Lai, "Theoretical analysis of Marangoni instability of an evaporating droplet by energy method," Int. J. Heat Mass Transfer 47, 3811 (2004).

[20] H. Machrafi, N. Sadoun, A. Rednikov, S. Dehaeck, P.C. Dauby, P. Colinet, "Evaporation rates and Bénard-Marangoni supercriticality levels for liquid layers under an inert gas flow," Microgravity Sci. Technol. 25, 251 (2013).

[21] H. Machrafi, A. Rednikov, P. Colinet, P.C. Dauby, "Bénard instabilities in a binary-liquid layer evaporating into an inert gas," J. Colloid Interface Sci. 349, 331 (2010).

[22] B. Sobac, P. Talbot, B. Haut, A. Rednikov, P. Colinet, "A comprehensive analysis of the evaporation of a liquid spherical drop", J. Colloid Interface Sci. 438, 306 (2015).

[23] H. Machrafi, A. Rednikov, P. Colinet, P.C. Dauby, "Time-dependent Marangoni-Bénard instability of an evaporating binary-liquid layer including gas transients," Phys. Fluids 25, 084106 (2013).

[24] H. Machraf, A. Rednikov, P. Colinet, P.C. Dauby, "Bénard instabilities in a binary-liquid layer evaporating into an inert gas: stability of quasi-stationary and time-dependent reference profiles," Eur. Phys. J. S. T. 192, 71 (2011).

[25] Nonetheless, to extend the validity of the analysis to the cases where the vapor is not very dilute (such as the case of the HFE-7100 liquid considered here as an example), we shall also employ a semi-heuristic Stefan-Fuchs correction [26].

[26] N.A Fuchs, R.S. Bradley, Vaporisation and Droplet Growth in Gaseous Media (Pergamon Press, Oxford, 1959).

[27] Note that keeping the convective contribution in (9) in spite of disregarding it in the bulk, where just the Laplace equations are solved for $\chi_{g, r e f}$ and $T_{g, r e f}$, constitutes what was referred to before as the Stefan-Fuchs correction. In this way, we end up with having $\chi_{g \Sigma}, r e f$ in the denominator of (11), whereas within a consistent dilute-vapor approach it should be disregarded there. 
[28] For simplicity, we have not applied the linearization to the denominator of (10) or (11), the error of which must anyway be comparable with that of the Stefan-Fuchs correction itself.

[29] B. Trouette, E. Chénier, F. Doumenc, C. Delcarte, B. Guerrier, Transient Rayleigh-BénardMarangoni solutal convection," Phys. Fluids 24, 074108 (2012).

[30] R.B. Bird, W.E. Steward, E.N. Lightfoot, Transport Phenomena (John Wiley \& Sons, New York, 2007).

[31] A.Y. Rednikov, Y.S. Ryazantsev, M.G. Velarde, "Active drops and drop motions due to nonequilibrium phenomena," J. Non-Equil. Thermody. 19, 95 (1994). 